\title{
Study on the construction of recombined plasmid pMG36e-lacc1 and the electroporation of Lactobacillus buchneri
}

\author{
Dan Xue, Ri Na*, Jiu Feng Guo and Teng Liu \\ Key Laboratory of Ion Beam Bioengineering, Inner Mongolia University, Hohhot, China
}

\begin{abstract}
With a goal of obtaining the engineering probiotics that can produce both lactic acid and laccase, Pleurotus eryngii was selected as the test material. First, the laccase gene (Lacc1) was cloned by using RT-PCR (the length of which is 1596bp), and then, the gene was ligated to the food-grade vector pMG36e from Lactobacillus buchneri. The recombinant expression vector pMG36e-Lacc1 was constructed by transforming it into Lactobacillus buchneri via an electroporation method. The recombinant plasmid was constructed successfully as confirmed by gel electrophoresis. Then, the optimum conditions for electroporation were determined. The research revealed that when the electric field intensity is $1.75 \mathrm{kV}$ with an SMRS medium recovery for $1.5 \mathrm{~h}$, the electroporation translation efficiency reaches its maximum level.
\end{abstract}

Keywords: Laccase, Lactobacillus buchneri, expression vector system

\section{Introduction}

Silage is a kind of feed that uses fresh green plant as the raw material; the quality of the feed remains relatively stable by using lactobacillus fementation [1]. In fact, lactobacillus is the key factor in determining the quality of the feed. An ideal strain should be able to more quickly produce a greater amount of acid; however, this ideal strain has not yet been realized [2]. As the animal husbandry industry has rapidly developed, it becomes more necessary to improve silage quality through improving the silage's utilization rate [3].

However, laccase, a kind of copper polyphenol oxidase, may be key to improving silage's utilization rate [4]. Laccase possesses a strong ability to degrade lignin so that the degradation of cellulose can be fully achieved. In recent years, the function of laccase degradation has attracted greater attention. As molecular biology has further developed, a variety of laccase genes have been successfully cloned, and thus, their heterologous expression has been implemented [5] . In this paper, Lactobacillus buchneri was used as the experiment material. The optimal conditions were explored by using electroporation techniques to introduce genes into Lactobacillus buchneri with the intention of building a new lactobacillus that has both lactate and laccase metabolic products. With this new strain, the quality of the feed would be greatly enhanced.

*Corresponding author: Ri Na, Key Laboratory of Ion Beam Bioengineering, Inner Mongolia University, Hohhot, China. Tel.:13171079869; Fax: 0471-4993139; E-mail: guojiufeng@imu.edu.cn. 


\section{Materials and methods}

\subsection{Bacterial strains and plasmids}

The Lactobacillus buchneri SYL from the silage that was used in the experiments is a strain that was isolated from Jia's Camp Ranch located in Inner Mongolia, China [6]. Escherichia coli TOP10 was purchased from the TIANGEN Corporation. The $P$. eryngii strains that were used in this study were collected from the Biotechnology Research Center of the Inner Mongolia Academy of Agricultural \& Animal Husbandry Sciences. Plasmid pMG36e (from the Key Laboratory of Ion Beam Bioengineering at Inner Mongolia University) is $3.6 \mathrm{~kb}$ in size, which is useful for transferring genes between Escherichia coli and the Lactobacillus species. It contains the pWV01 origin of replication, a promoter $\mathrm{p} 32$, polyclonal loci (MCS), the repA gene, and an erythromycin resistance gene, which has been previously reported to be functional in a wide range of bacteria [7].

\subsection{Measuring method}

\subsubsection{Laccl gene amplification}

The Laccl gene was amplified by RT-PCR as previously described by Dan Xue et al. [8].

\subsubsection{Construction of recombinant plasmids pMG36e- Lacc1}

Both the 1600bp fragment of recombinant pGM-T and vector pMG36e were digested with Sph I and Sac I (TaKaRa Biotechnology Co., Ltd. Dalian, China) and then ligated by T4 ligase (TakaRa Biotechnology Co., Ltd. Dalian, China). Next, the recombinant plasmid was introduced into E. coli TOP10, and then, the bacteria were spread onto LB agar plates containing erythromycin. After growth occurred, single colonies were picked and grown in LB liquid medium that contained erythromycin. The Laccl gene was PCR amplified using specific primers. The recombinant plasmid pMG36e-Lacc1 was constructed.

\subsubsection{The erythromycin susceptibility test and the preparation of electrocompetent cells}

A Lactobacillus buchneri strain that had been preserved in the laboratory was activated according to reference [9]. The strains that produced the greatest acid amounts were selected. The competent cells were prepared, and the appropriate concentration of erythromycin was determined.

\subsubsection{Electroporation conditions}

Electroporation was carried out using the Eppendorf Electroporator 2510. Electroporation cuvettes $(0.1 \mathrm{~cm})$ were chilled on ice, and $50 \mu \mathrm{L}$ of competent cells were mixed on ice with $5 \mu \mathrm{L}$ of recombinant plasmids pMG36e- Lacc1. The mixture was mixed well and then allowed to sit on ice for $10 \mathrm{~min}$. The mixture of cells and DNA was transferred to the bottom of cold $0.1-\mathrm{cm}$ electroporation cuvettes and then electroporated at $0.0 \mathrm{kV}, 1.5 \mathrm{kV}, 1.75 \mathrm{kV}, 2.0 \mathrm{kV}, 2.25 \mathrm{kV}$, and $2.5 \mathrm{kV}$, respectively. Then, $950 \mu \mathrm{L}$ of MRS or SMRS medium was immediately added after the pulse application. The entire cell suspension was transferred into a $1.5 \mathrm{~mL}$ tube and incubated at $37^{\circ} \mathrm{C}$ for $1.5 \mathrm{~h}, 2.0 \mathrm{~h}$, and $2.5 \mathrm{~h}$. Each experiment was repeated three times, and dilutions were spread ten times onto the MRS agar media containing $0.5 \mu \mathrm{g} / \mathrm{mL}$ erythromycin. The plates were grown at $37^{\circ} \mathrm{C}$ for $48 \mathrm{~h}$. The transformation efficiency (cfu/ $\mu \mathrm{gDNA})$ was calculated by the following equation: Transformation efficiency $(c f u / \mu g$ DNA) $=$ Appropriate dilutions $\times$ Colony count. 


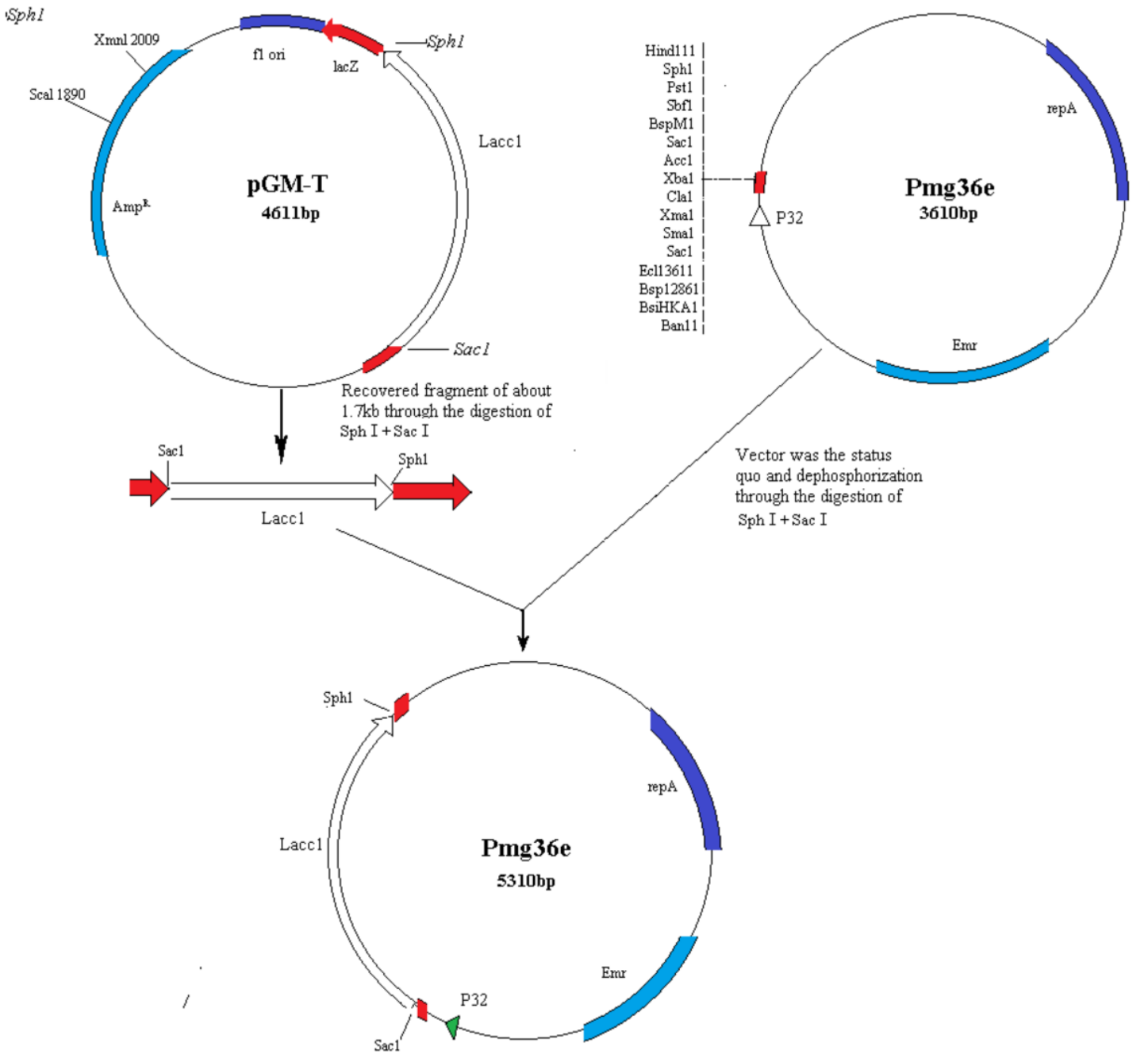

Fig. 1. Construction of recombinant expression vector pMG36e-Lacc1.

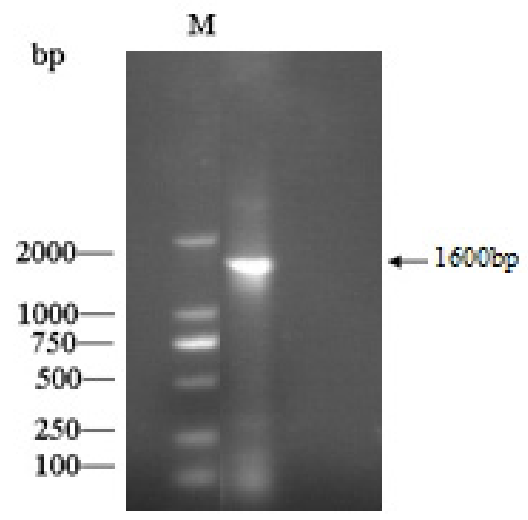

Fig. 2. Identification of recombinant plasmid pMG36e-Lacc1 by diagnostic PCR. 


\subsubsection{PCR identification of Lactobacillus's recombinant plasmid}

The white colonies were picked directly from the MRS plates that contained erythromycin, and then, PCR was performed. The Laccl gene was PCR amplified from the recombinant plasmid pMG36eLacc1. The PCR reaction system was as follows: $10 \mu \mathrm{L}$ of 2Taq PCR Master Mix, $1 \mu \mathrm{L}$ of primer P $(10 \mathrm{pmol} / \mu \mathrm{L}), 1 \mu \mathrm{L}$ of prime, Q $(10 \mathrm{pmol} / \mu \mathrm{L})$, and $8 \mu \mathrm{L}$ of RNase-free water for a total volume of 20 $\mu \mathrm{L}$. The PCR conditions were as follows: $93^{\circ} \mathrm{C}$ for $3 \mathrm{~min} ; 35$ cycles of $93^{\circ} \mathrm{C}$ for $15 \mathrm{~s}, 63^{\circ} \mathrm{C}$ for $30 \mathrm{~s}$, and $68^{\circ} \mathrm{C}$ for $1.5 \mathrm{~min}$; and then, indefinite at $4^{\circ} \mathrm{C}$. Identification was confirmed using $1.0 \%$ agarose electrophoresis. Since the correct $1600 \mathrm{bp}$ fragment was amplified, the results confirmed that the recombinant plasmid was successfully transformed into Lactobacillus Buchneri through electroporation.

\section{Results and Analysis}

\subsection{Cloning and sequencing of Lacc1}

The PCR product was a DNA fragment of $1600 \mathrm{bp}$, the sequencing results verified that the PCR product was $1756 \mathrm{bp}$. Through DNAMAN analysis, the following data was obtained: the position of Laccl's complete open reading frame in $76 \sim 1672$ bp (1596 bp in length), start codon ATG, and the termination codon TAG. The Laccl gene open reading frame is composed of 1596 nucleotides, which encodes a polypeptide of 531 amino acids, as detailed in references [8].

\subsection{Construction of recombinant expression vector of Lactobacillus}

The recombinant pGM-T and vector pMG36e were digested with Sph I and Sac I after the gel recycling and then ligated using T4 DNA ligase, leading to the construction of pMG36e-Lacc1. The construction procedure is outlined in Figure 1. The full length of the $1600 \mathrm{bp}$ fragment was amplified with specific primers as shown in Figure 2. Furthermore, by sequencing, the length of the $1596 \mathrm{bp}$ segment was obtained, in accordance with the length of Laccl. This confirmed that the recombinant expression plasmid pMG36e-Lacc1 has been successfully constructed.

\subsection{The sensitivity of the Lactobacillus buchneri to erythromycin}

When the erythromycin concentration is $0.5 \mu \mathrm{g} / \mathrm{mL}$ in the MRS medium, it can inhibit Lactobacillus growth. Accordingly, no colonies grew on a plate when the erythromycin concentration in the wells ranged from $0.5 \mu \mathrm{g} / \mathrm{mL}$ to $5 \mu \mathrm{g} / \mathrm{mL}$. Therefore, a $0.5 \mu \mathrm{g} / \mathrm{mL}$ erythromycin concentration was selected as the minimum inhibitory concentration.

\subsection{Electroporation conditions of Lactobacillus buchneri}

As more research has been conducted on the molecular biology of Lactobacillus, there has been a series of cloning vectors and expression vectors based upon a Lactobacillus plasmid. The advantage of the lactobacillus expression system is favorable compared with that of the E. coli non- food -grade expression system. At present, protoplast transformation and electroporation are the main transformation methods for Lactobacillus. Protoplast transformation, however, has many unwanted constraints: it is time-consuming, has a low efficiency, and has a poor stability [10]. Thus, electroporation is widely used in Lactobacillus [11]. 


\subsubsection{Effects of field strength on transformation efficiency}

Different field strengths were selected for the electrotransformation. The recovered bacteria liquid was spread onto MRS agar media containing erythromycin. The varying transformation efficiency under different voltages and field strengths is shown in Figure 3.

As shown in Figure 3, the transformation efficiency initially increases as the electric field strength increases. When the field strength was $1.75 \mathrm{kV}$, the transformation efficiency reached its maximum: $2500 \mathrm{cfu} / \mu \mathrm{g}$ of DNA. However, as the field strength continued to increase to greater than $1.75 \mathrm{kV}$, the electrotransformation efficiency started to decrease.

\subsubsection{Effects of recovery medium and time periods on transformation efficiency}

In order to explore how the recovery medium and time periods would affect transformation efficiency, MRS (the Lactobacillus culture medium) and SMRS (MRS contains a certain amount of sugar) media were used for the recovery, and the culture condition was $37^{\circ} \mathrm{C}$ for $1.5 \mathrm{~h}, 2.0 \mathrm{~h}$, and $2.5 \mathrm{~h}$. The results are shown in Figure 4.

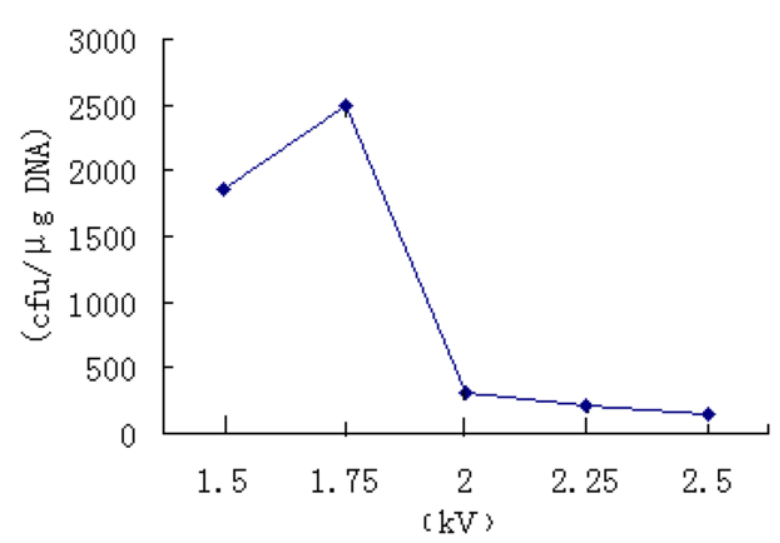

Fig. 3. Effects of electric field strength on electrotransformation efficiency.

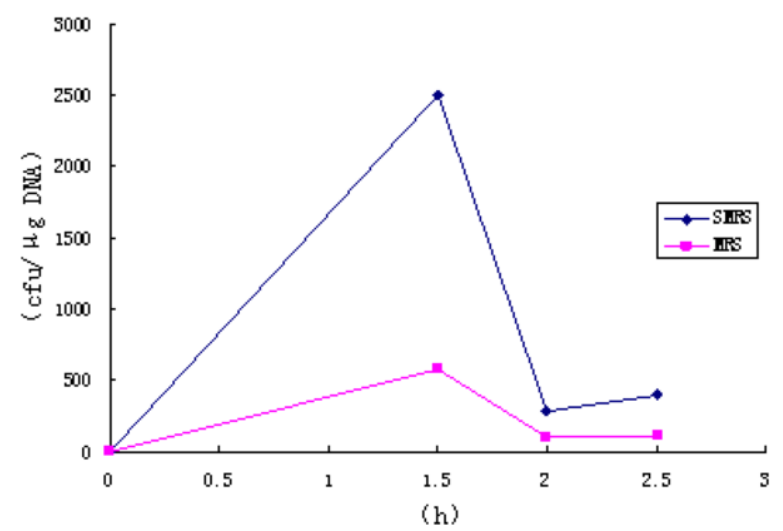

Fig. 4. Effects of recovery medium and time periods on transformation efficiency.

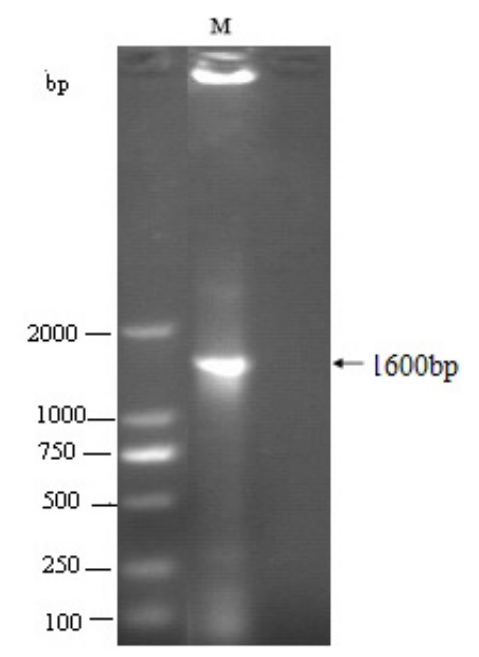

Fig. 5. The identification of recombinant plasmid in Lactobacillus. 
As shown in Figure 4, an approximately fivefold increase in efficiency was observed when the SMRS medium was used for the recovery of the electroporated cells instead of the MRS medium. The highest transformation efficiency was obtained at a recovery time of $1.5 \mathrm{~h}$, but further extending the incubation recovery results in a decline in electrical stimulation efficiency.

\subsection{Detection of recombined Lactobacillus plasmid by PCR}

The white colonies that had been selected from the MRS plates that contained erythromycin were detected by PCR, and the length of the $1600 \mathrm{bp}$ fragment was amplified. The colony PCR results confirmed that the recombinant expression plasmid pMG36e-Lacc1 has been successfully transformed into Lactobacillus buchneri. The PCR results are shown in Figure 5.

\section{Discussion}

Lignin and hemicellulose bind covalently to form complex natural polymers with an amorphous structure. The cellulose molecules are embedded in these polymers so that lignin cannot be digested by animals because there are no lignin degradation enzymes present in sheep and other animals. In order to make full use of straw, the lignin degradation must be solved [12]. Currently, white rot fungi is the main source of laccase [13]; at the same time, edible fungus is a green food, and the application of laccase that comes from edible fungi can ensure the safety of feed [14].

A study determined that the Pleurotus eryngii laccase enzyme activity is the highest, so it was selected as the test material for cloning the laccase gene. Additionally, Lactobacillus is a thicker cell wall, Gram-positive bacteria [15], so it is difficult for exogenous DNA to enter into the host cell. Thus, electroporation is commonly used to improve the transformation efficiency of Lactobacillus.

This paper primarily explored the optimum electroporation conditions of Lactobacillus buchneri that was isolated from silage, through the transformation of the laccase gene (Laccl) connected with the food-grade vector pMG36e from lactobacillus buchneri. The recombinant expression vector pMG36e-Lacc1 was constructed by using the electroporation method to transform the vector into lactobacillus buchneri.

Then, conditions that affected the electroporation efficiency (electric field strength, recovery medium and time periods) were evaluated to determine the optimal conditions. For example, after exploring the effect that field strength had on transformation efficiency, data revealed that the transformation efficiency initially increased as the electric field strength increased, ultimately reaching a maximum efficiency of $2500 \mathrm{cfu} / \mu \mathrm{g}$ of DNA at $1.75 \mathrm{kV}$. However, after this point, as the field strength continued to increase, the electrotransformation efficiency started to decrease instead. Next, from exploring the effects of recovery medium and time periods on transformation efficiency, data indicated that there was an approximately fivefold increase in efficiency observed when the SMRS medium was used for the recovery of the electroporated cells instead of the MRS medium. When the recovery time was $1.5 \mathrm{~h}$, the transformation efficiency reached its maximum; however, as the incubation time of recovery was further extended, the electrical stimulation efficiency began to decline. This is because with the extension culture by recovery medium (without antibiotics), Lactobacillus with a transfer plasmid and without the transfer plasmid were in the propagation process. The Lactobacillus without the transfer plasmid was breeding faster, and the plasmid-containing Lactobacillus perhaps lost the plasmid in the propagation process. Therefore, it is possible to conclude that that plasmid-containing Lactobacillus dwindled as time passed. This resulted in a decrease in the 
number of colonies that grew on the MRS agar media that contained antibiotics. Then, as previously mentioned, as the incubation time of recovery was further extended, the electrical stimulation efficiency declined.

In this paper, the recombinant expression vector pMG36e-Lacc1 was successfully constructed by using an electroporation method to transform the plasmid into Lactobacillus buchneri. This will lay the foundation for the preparation of probiotic agents as well as obtain the engineering probiotics than can produce both lactic acid and laccase.

\section{References}

[1] W.J. Zhang, J.Q. Wang, Y.S. Gong et al., The research progress of straw feed resources development and utilization, Animal Science Abroad 3 (2001), 15-18.

[2] X.J. Xi, L.J. Han, H.A.R.A. Shin-ichiro et al., Effects of lactobacillus and cellula se on the quality of corn stover silage, Journal of China Agricultural University 2 (2003), 21-24.

[3] X.J. Xi, Effeets of activities on the quality of corn stover silage, PhD dissertation, China Agricultural University, 2002.

[4] B. Reinhammar, Volume II, In: Copper Proteins and Cooper Enzymes, R. Lontie, ed., CRC Press, Boca Raton, 1984.

[5] H.Y. Xu, L.L. Zhang, F.E. Wang et al., The research progress of White-rot fungi, Chinese Journal of Animal Husbandry and Veterinary Medicine 12 (2007), 11-12.

[6] G.N. Ba, Research on the effect of corona electric field treatment on the characteristics of lactic acid bacteria silage, $\mathrm{PhD}$ dissertation, Inner Mongolia University, 2012.

[7] G. Vander, V. Vander and J. Kok, Construction of lactocoual expression vector expression of hen egg white lysozyme in Lactococcus lactis, Appl. Environ. Microbiol. subsp. 55 (1989), 224-228.

[8] D. Xue, J.F. Guo, R. Na et al., Cloning and sequence analysis of laccase gene DNA from pleurotus eryngii, Edible and Medicinal Mushrooms 5 (2013), 282-287.

[9] H.M. Guo, X.X. Gu, Y.L. Zhang et al., Research of electrotransformation processing of lactobacilli delbrueckii subsp. bulgaricus with the constitutive expression vector pMG36e, Journal of Chinese Institute of Food Science and Technology 2 (2011), 8-16.

[10] B.M. Chassy and J.L. Flickinger, Transformation of Lactobacillus casei by electroporation, FEMS Microbiol. Letts. 44 (1987), 173-177.

[11] I.B. Powell and M.G. Achen, Asimple and rapid method forgenetic transformation of Lactic streptococci by electroporation, Appl. Environ. Microbiol. 54 (1988), 655-660.

[12] Y.L. Sun, Study on production of substances eliminating free radicals during lignocellulose degradation by white rot fungi, PhD dissertation, Huazhong University of Science \& Technology, 2005.

[13] J.P. Lin, W. Lian, L.M. Xi, et al., Production of laccase by Coriolus versicolor and its application in decolorization of dyestuffs, Journal of Environmental Sciences 15 (2003), 1-4.

[14] J. Pan, K.J. Shun, C. He et al., Utilization of edible fungi on crop straw bioderadation into animal feed, Acta Ecologiae Animalis Domastici 2 (2011), 85-91.

[15] B. H. Guo, Probiotics, Chemical Industry Press, Beijing, 2004, pp. 30-32. 